\title{
ANÁLISE DE DECOMPOSIÇÃO DA PROJEÇÃO DE CONSUMO DE ENERGIA ELÉTRICA NO BRASIL PARA O SETOR RESIDENCIAL
}

Fabrício V. Andrade ${ }^{1}$

Ricardo B. Pinheiro ${ }^{2}$

\begin{abstract}
Resumo: Neste trabalho é usada a técnica de decomposição Logarithmic Mean Divisia Index (LMDI) para avaliar as variações de consumo e as projeções do consumo de energia elétrica no setor residencial no Brasil para o período 2005-2020. Nesta análise, o consumo de energia elétrica foi desagregado em quatro efeitos: Atividade, Estrutura, Posse e Intensidade. Para cada categoria de uso final foram avaliados estes efeitos em períodos distintos e, desta forma, foi possível estimar como cada categoria e seus respectivos equipamentos, a quantidade de consumidores e a posse contribuíram para a variação no consumo de eletricidade no setor residencial. Dentre vários resultados, concluiu-se que o consumo de energia elétrica deverá alcançar 177 TWh em 2020, um aumento de $110 \%$ em relação a 2005. Também, percebe-se que grande parcela do aumento do consumo de energia elétrica será devido ao efeito Atividade, que está associada ao aumento do número de domicílios ligados a rede de energia elétrica. No período de 2005-2020, o efeito Atividade deverá ser responsável por 57\% do aumento de consumo de energia elétrica no setor residencial.
\end{abstract}

Palavras-Chave: Consumo de energia elétrica; setor residencial; projeção; LMDI.

Abstract: This work aimed to apply decomposition techniques called Logarithmic Mean Divisia Index (LMDI) to evaluate the variation of electricity consumption and its forecast for the residential sector for the period 2005-2020. Electricity consumption was decomposed into four effects/indicators: Activity, Structure, Intensity and Ownership. From the decomposition, it was possible to estimate how each category and its related appliances, the number of consumers and the ownership, have contributed to the variation of electricity consumption in the residential sector for the period 2005-2020. Among other results, we may conclude that the electricity consumption in the residential sector may reach $177 \mathrm{TWh}$ in 2020. Indeed, we realize that a great amount of the rising of consumption is due to the Activity effect, which is closely related to the increase of the number of households. We may also consider that from 2005 to 2020, the Activity effect may be responsible for 57 percent of the increase of electricity consumption in the residential sector.

Keywords: Electrical energy consumption, residential sector, forecast, LMDI

\footnotetext{
${ }^{1}$ DEN/UFMG - Departamento de Engenharia Nuclear/ Universidade Federal de Minas Gerais. E-mail: fabricioenergy@yahoo.com

${ }^{2}$ DEN/UFMG - Departamento de Engenharia Nuclear/ Universidade Federal de Minas Gerais. E-mail: rbp@nuclear.ufmg.br

ENGEVISTA, V. 16, n. 4, p.340-355, Dezembro 2014 


\section{Introdução}

O setor residencial é um dos principais responsáveis pelo consumo de energia elétrica no Brasil. Segundo o último Balanço Energético Nacional, BEN 2012 (Brasil, 2012a), o setor residencial foi responsável pelo consumo de $23,6 \%$ da energia elétrica consumida em 2011, ficando atrás apenas do setor industrial, com 43,6\%.

Depois do racionamento de energia elétrica, em 2001, percebeu-se que o consumo das famílias tem-se elevado significativamente, retomando a trajetória de crescimento de antes da crise. Somente nos últimos anos, de 2005 a 2011, o consumo neste setor, em média, cresceu $5,1 \%$ ao ano. Dentre os principais motivos desta variação de consumo, podem-se citar: a melhoria da renda das famílias, aquisição de novos equipamentos eletroeletrônicos e mudanças de hábitos de consumo que podem estar associados à política de ampliação ao acesso da energia elétrica e das políticas sociais de distribuição de renda.

Com propósito de analisar essas mudanças no consumo de energia elétrica no setor residencial, foi utilizada neste artigo, a técnica de decomposição Logarithmic Mean Divisia Index (LMDI) para avaliar as variações de consumo e das projeções de consumo de energia elétrica no setor residencial a partir da quantificação dos efeitos de Atividade, Estrutura, Intensidade e Posse. Neste caso, entenda-se Atividade, como a quantidade de domicílios; Estrutura, como a porcentagem de domicílio em um dado estrato de renda; Intensidade, como o consumo de energia de um dado serviço por equipamento; e Posse, como a quantidade de um dado equipamento por domicílio consumidor. Para tanto, foram considerados sete categorias de uso final, seus respectivos consumos de energia elétrica no ano de 2005 e 2010, e também suas projeções para 2015 e 2020.
Portanto, o principal objetivo deste artigo consiste em explicar a variação de consumo de energia elétrica no setor residencial em termos dos efeitos Atividade, Estrutura, Posse e Intensidade para os diversos equipamentos eletroeletrônicos existentes nos lares brasileiros.

\section{0 setor residencial brasileiro e $o$ consumo de energia elétrica}

O consumo de energia elétrica no Brasil, em 2011, correspondeu a 480 TWh, sendo que o setor residencial foi responsável por 112 TWh (Brasil, 2012a). Conforme pode ser visto na Figura 1, a energia elétrica é o principal insumo energético consumido no setor, ultrapassando a lenha, que foi por muitos anos o energético mais consumido.

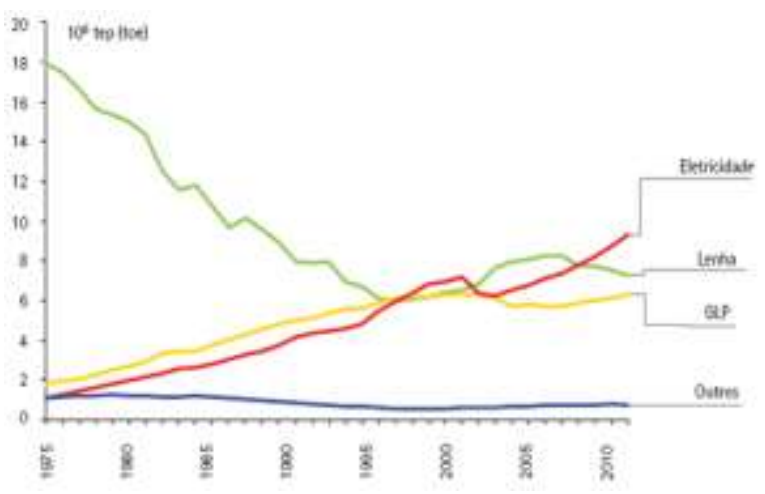

Figura 1: Evolução do consumo de energia final para o setor residencial, 1975-2011.

Fonte: BEN 2012 (Brasil, 2012a).

Com relação aos usos finais, é possível dizer que a energia elétrica é utilizada principalmente para o condicionamento de alimentos, para o aquecimento de água e iluminação, representando somente essas três categorias $76 \%$ de toda energia elétrica consumida, em 2010, no setor residencial (Brasil, 2011). 


\subsection{A estrutura de consumo}

A elaboração da estrutura de consumo no setor residencial brasileiro desagregada por equipamento foi baseada na Pesquisa de posse de equipamentos $e$ hábitos de uso, no setor residencial, realizada pelo Procel/Eletrobrás (2007). Nesta pesquisa foi feito um levantamento detalhado da posse e hábitos de uso dos principais eletrodomésticos no ano de 2005 para o Brasil, sendo que para tanto foi necessária a aplicação de quase 10.000 questionários e selecionados mais de 4.300 como amostras.

A partir desta pesquisa, é possível estimar o consumo de energia elétrica por tipo de equipamento e também a posse média desse equipamento por domicílio. $\mathrm{Na}$ Tabela 1 são apresentados os consumos específicos para diversos equipamentos eletroeletrônicos no ano de 2005, desagregada por três estratos de renda.

Tabela 1 - Consumo específico (kWh/ano) do estoque de equipamentos em 2005

\begin{tabular}{lccc}
\hline \multicolumn{1}{c}{ EQUIPAMENTO } & Até 3SM & $\begin{array}{c}\text { Entre } \\
\text { 3SM } \\
\text { e }\end{array}$ & $\begin{array}{c}\text { Maior } \\
\text { que } \\
\text { 7SM }\end{array}$ \\
\hline Chuveiro elétrico & 532 & 724 & 912 \\
Geladeira & 398 & 455 & 534 \\
\hline Ar condicionado & 257 & 424 & 481 \\
\hline Lâmpada incandescente & 50 & 55 & 86 \\
\hline Lâmpada fluorescente & 19 & 18 & 28 \\
\hline Freezer & 346 & 395 & 523 \\
\hline Aparelho de Televisão & 97 & 112 & 117 \\
\hline M. Lava roupa & 12 & 13 & 15 \\
\hline Micro-ondas & 60 & 68 & 83 \\
\hline Ferro de passar roupa & 65 & 81 & 96 \\
\hline Aparelho de som & 44 & 47 & 55 \\
Computador & 21 & 30 & 49 \\
\hline Ventilador de mesa & 29 & 39 & 45 \\
\hline Ventilador de teto & 29 & 40 & 46 \\
\hline
\end{tabular}

Nota: * SM- salário mínimo. Fonte:

Elaboração própria a partir de Procel /Eletrobrás (2007; 2011)

Além do consumo específico, a posse de equipamentos, a renda e a quantidade de domicílios são variáveis importantes que também estão relacionadas diretamente com o consumo de eletricidade. Em princípio, quanto maior a posse, maior a renda e o número de domicílios, maior será o consumo de energia elétrica.

Segundo a Pesquisa Nacional por Amostra de Domicílios - PNAD 2009 (IBGE, 2009), havia no Brasil 58,6 milhões de domicílios, em 2009, sendo que $99 \%$ desses domicílios possuíam energia elétrica. Desses domicílios que possuíam energia elétrica, infelizmente, nem todos são consumidores legalmente atendidos pelas concessionárias brasileiras, como aponta Araujo (2007). Segundo ele, em 2005, $15 \%$ da energia elétrica demandada no setor residencial foi perdida na distribuição, sendo que $32 \%$ desta energia (4,8\% do total) foram consideradas perda não técnica, ou seja, desvios de energia ou erros associados à má gestão de faturamento de consumo de energia nos domicílios. Comparando o número de domicílios eletrificados no Brasil, em 2005, com o número de consumidores declarados pelas concessionárias no mesmo ano (Achão, 2009), corresponderia a aproximadamente $5,5 \%$ dos domicílios brasileiros não pagando energia elétrica. Dentre as regiões brasileiras, as regiões Norte e Nordeste são as que apresentam maiores índices de perda não técnica de energia.

Já a posse de equipamentos no setor residencial brasileiro pode ser estimada baseando-se, também, na pesquisa desenvolvida pelo Procel/Eletrobrás (2007). Na Tabela 2 é mostrada a posse média por domicílio em 2005, desagregado por três estratos de renda, para diversos equipamentos. Entenda-se posse como a quantidade de um dado equipamento por domicílio consumidor.

Tabela 2 - Quantidade média de equipamentos eletroeletrônicos por domicilio no Brasil, em 2005 


\begin{tabular}{lccc}
\hline \multicolumn{1}{c}{ EQUIPAMENTO } & Até 3SM & $\begin{array}{c}\text { Entre } \\
\text { 3SM } \\
\text { e }\end{array}$ & $\begin{array}{c}\text { Maior } \\
\text { que } \\
\text { 7SM }\end{array}$ \\
\hline Chuveiro elétrico & 0,57 & 0,81 & 0,82 \\
\hline Geladeira & 0,92 & 1,02 & 1,08 \\
Ar condicionado & 0,02 & 0,09 & 0,42 \\
Lâmpada incandescente & 4,08 & 4,82 & 6,10 \\
Lâmpada fluorescente & 1,66 & 3,22 & 6,30 \\
Freezer & 0,09 & 0,21 & 0,48 \\
Aparelho de Televisão & 1,07 & 1,37 & 2,02 \\
\hline M. Lava roupa & 0,39 & 0,73 & 0,88 \\
Micro-ondas & 0,07 & 0,32 & 0,66 \\
\hline Ferro de passar roupa & 0,84 & 0,96 & 0,98 \\
Aparelho de som & 0,53 & 0,74 & 1,00 \\
Computador & 0,04 & 0,17 & 0,60 \\
\hline Ventilador de mesa & 0,61 & 0,81 & 0,92 \\
\hline Ventilador de teto & 0,16 & 0,48 & 0,72 \\
\hline
\end{tabular}

Fonte: Elaboração própria a partir de

Procel/Eletrobrás (2007)

Destaca-se, na Tabela 2, a significativa variação de posse de equipamentos eletroeletrônicos por estrato de renda. Conforme se pode notar, a posse de aparelhos como ar condicionado e freezer chega a ser cinco vezes maior entre aqueles domicílios de maior renda, em comparação com aqueles de menor renda. Além disso, não se pode esquecer que o clima é um fator determinante na composição da posse de equipamentos eletroeletrônicos nos lares brasileiros. Por exemplo, no caso do chuveiro elétrico a posse desse equipamento na Região Norte, em média, é 0,05 chuveiros por domicílio, enquanto na Região Sul chega a 1,17 chuveiros por domicílio (Procel/Eletrobrás, 2007).

Para facilitar a apresentação do consumo de energia elétrica é útil organizar os equipamentos em categorias de uso final. Portanto, o consumo de energia elétrica no setor residencial pode ser dividido em sete categorias de usos finais: Aquecimento de água, Condicionamento de alimentos, Condicionamento de ambientes, Iluminação, Serviço, Lazer e Demais equipamentos, sendo que cada equipamento eletroeletrônico pode ser associado a estes usos finais, como se segue: chuveiro, associado à categoria de Aquecimento de água; geladeira e freezer, associados à categoria de Condicionamento de alimentos; ar condicionado e ventilador, associados à categoria de Condicionamento de ambientes; lâmpadas, associadas à categoria de Iluminação; máquina de lavar roupa, micro-ondas e ferro de passar, associados à categoria de Serviço; computador, aparelho de som, aparelho de televisão, que são associados à categoria de Lazer e, por fim, todos os demais equipamentos existentes nos domicílios foram incluídos na categoria Demais equipamentos. Desta forma, na Tabela 3 é apresentado o consumo de energia elétrica no setor residencial para o Brasil, por categoria de uso final, para os três estratos de renda, em 2005.

Tabela 3 - Consumo de energia elétrica no setor residencial, por categoria, em 2005 (TWh/ano)

\begin{tabular}{ccccc}
\hline Categorias & $\begin{array}{c}\text { Até } \\
\text { 3SM }\end{array}$ & $\begin{array}{c}\text { Entre } \\
\mathbf{3 M ~ e} \\
\mathbf{7 S M}\end{array}$ & $\begin{array}{c}\text { Maior } \\
\text { que } \\
\mathbf{7 S M}\end{array}$ & Total \\
\hline Aquecimento de & 7,8 & 8,4 & 6,4 & 22,6 \\
água & $(26 \%)$ & $(31 \%)$ & $(24 \%)$ & $(27 \%)$ \\
Condicionamento & 10,2 & 7,9 & 7,1 & 25,1 \\
de alimentos & $(34 \%)$ & $(29 \%)$ & $(26 \%)$ & $(30 \%)$ \\
Condicionamento & 0,7 & 1,3 & 2,4 & 4,3 \\
de ambientes & $(2 \%)$ & $(5 \%)$ & $(9 \%)$ & $(5 \%)$ \\
Iluminação & 6,1 & 4,6 & 6,0 & 16,7 \\
& $(20 \%)$ & $(17 \%)$ & $(22 \%)$ & $(20 \%)$ \\
Serviço & 1,6 & 1,6 & 1,4 & 4,6 \\
& $(5 \%)$ & $(6 \%)$ & $(5 \%)$ & $(5 \%)$ \\
Lazer & 3,3 & 2,8 & 2,7 & 8,8 \\
& $(11 \%)$ & $(10 \%)$ & $(10 \%)$ & $(10 \%)$ \\
Demais & 0,4 & 0,6 & 0,9 & 1,9 \\
equipamentos & $(1 \%)$ & $(2 \%)$ & $(3 \%)$ & $(2 \%)$ \\
Total & $\mathbf{3 0 , 2}$ & $\mathbf{2 7 , 1}$ & $\mathbf{2 6 , 7}$ & $\mathbf{8 4 , 1}$ \\
& $(\mathbf{1 0 0 \% )}$ & $(\mathbf{1 0 0 \% )}$ & $(\mathbf{1 0 0 \% )}$ & $(\mathbf{1 0 0 \% )}$ \\
\hline
\end{tabular}

\section{Metodologia}

Para alcançar o objetivo deste artigo, que consiste em explicar a variação de consumo de energia elétrica no setor residencial em termos da evolução do número de domicílios com acesso à energia elétrica no Brasil, da evolução da distribuição de renda, da variação do estoque de equipamentos eletroeletrônicos e da variação da eficiência de consumo de energia por cada equipamento, foi projetado $\mathrm{o}$ consumo de energia elétrica por equipamento até 2020 e, posteriormente, utilizada uma metodologia de análise de decomposição de fatores que permite explicar o consumo total a partir dos seus efeitos. A forma como foi elaborada a projeção de consumo e a descrição da 
metodologia de análise de decomposição estão apresentadas a seguir.

\subsection{Projeção de consumo}

Considerando o consumo de energia elétrica no setor residencial a partir de 2005, devido à disponibilidade de dados encontrados na Pesquisa de posse de equipamentos e hábitos de uso, no setor residencial, foi estimado $\mathrm{o}$ consumo para 2010 e projetado o consumo para 2020.

Para fazer a projeção de consumo foi considerado, como uma das variáveis fundamentais, o nível da atividade econômica doméstica, que pode ser medido pelo crescimento do PIB. Este indicador apresentou um valor médio histórico de 4,3\% ao ano, no período de 2005-2010 e uma projeção de 5,0\% ao ano no período de 2010-2020 (Brasil, 2011). Além disso, o número de domicílios consumidores aumentou, em média, $3,2 \%$ ao ano no período de 2005 2010 e espera-se que cresça a uma taxa de $3,6 \%$ e $2,2 \%$ ao ano, no período de 2010 2015 e 2015-2020, respectivamente, chegando ao fim da projeção com 75,7 milhões de domicílios (Brasil, 2011). Ademais, as principais hipóteses socioeconômicas são apresentadas a seguir:

Redução do número de pessoas por domicílio, a partir de um ajuste logístico dos dados históricos, disponibilizados pelo IBGE (2012). Na Tabela 4, essas projeções estão apresentadas.

Tabela 4 - Estimativas e projeções para o número de pessoas por domicílio para os três estratos de renda

\begin{tabular}{ccccc}
\hline Renda & $\mathbf{2 0 0 5}$ & $\mathbf{2 0 1 0}$ & $\mathbf{2 0 1 5}$ & $\mathbf{2 0 2 0}$ \\
\hline Até 3SM & 3,3 & 3,0 & 2,8 & 2,7 \\
$\begin{array}{c}\text { Entre 3 e } \\
\text { 7SM }\end{array}$ & 3,7 & 3,4 & 3,3 & 3,2 \\
$\begin{array}{c}\text { Maior que } \\
\text { 7SM }\end{array}$ & 3,6 & 3,4 & 3,3 & 3,2 \\
\hline
\end{tabular}

ENGEVISTA, V. 16, n. 4, p.340-355, Dezembro 2014
Redução da proporção de domicílios, com renda até 3 salários mínimos (SM), de $53,0 \%$ para $40,9 \%$ no período 2005-2020; aumento da proporção de domicílios com renda acima de $7 \mathrm{SM}$, de $17,5 \%$ para $22,4 \%$, no mesmo período; e, aumento de $29,5 \%$ para $36,7 \%$ para os domicílios com renda entre 3 SM e 7 SM (Ernest \& Young Terco, 2008).

Para as demais hipóteses, inclusive as tecnológicas, as principais são:

Aquecimento de água: Aumento de potência de $0,48 \%$ ao ano, para os chuveiros elétricos (Brasil, 2011) e diminuição do tempo de uso do chuveiro elétrico, devido à redução do tamanho das famílias brasileiras. Demais variáveis relacionadas ao consumo específico do chuveiro foram mantidas constantes.

Durante o período de 2005-2010, a região Nordeste foi uma das principais responsáveis pelo aumento do número de domicílios no Brasil (IBGE, 2012). Como nesta região a posse de chuveiro elétrico é pequena, considerou-se que em 2010 a proporção de chuveiros no Brasil seria a média ponderada das proporções regionais pelo peso do número de domicílios por região.

Para depois de 2010, considerouse que o número de domicílios que teriam chuveiro elétrico no estrato de renda até 3 SM sofreria uma redução de $0,8 \%$ ao ano, chegando a $50 \%$ em 2020. Isto porque o programa "Minha Casa Minha Vida" (MCMV) do governo federal seria responsável por induzir a redução do número de chuveiros ao substituir este equipamento por aquecedores solares. No programa MCMV são previstos aproximadamente 1,44 milhões de casas, na fase 1 e 2, para este estrato de renda (neste caso, considera-se que $840 \mathrm{mil}$ seriam entregues até 2015, e, o restante, depois de 2015) (Brasil, 2012b). Para as famílias entre $3 \mathrm{SM}$ e $7 \mathrm{SM}$, foi considerado que o programa MCMV (fase 2) induziria uma penetração de $4,1 \%$ de residências com Sistemas de Aquecimento Solar (SAS) do total de 800 mil domicílios contratados, sendo que a 
primeira metade seria implementada até 2015 e a outra, até 2020 . Isto porque a taxa de $4,1 \%$ representa a quantidade de domicílios que possuíram o SAS de forma facultativa na primeira fase do programa MCMV, portanto, por hipótese, esta taxa foi utilizada também na segunda fase, para o estrato de renda entre $3 \mathrm{SM} \mathrm{e}$ 7 SM (Brasil, 2013). Finalmente, nos domicílios de renda acima de $7 \mathrm{SM}$ haveria uma penetração significativa de gás natural (GN), considerando-se, como hipótese, que todos os novos domicílios que aqueceriam água com GN estariam neste estrato de renda, havendo, dessa forma, uma maior substituição da energia elétrica e do GLP, como forma de aquecimento de água para banho. Diante disto, a posse de chuveiros elétricos reduziria em $0,2 \%$ ao ano até 2020, conforme nota técnica da EPE sobre eficiência energética na indústria e nas residências (Brasil, 2010a). Neste caso, a posse de chuveiro reduziria para $80 \%$ e o aquecedor a GN aumentaria para $9 \%$, em 2020, mantendo os demais sistemas de aquecimento de água constantes.

Condicionamento de alimentos: Espera-se melhoria da eficiência de refrigeradores e freezers por substituição de equipamentos velhos por novos, sendo que, em 2020, não haveria nenhum equipamento com eficiência abaixo da faixa B, estabelecida pelo Inmetro (2011). Nesta categoria, as geladeiras teriam uma redução de consumo de $0,8 \%$ ao ano, $0,2 \%$ ao ano e $1,3 \%$ ao ano, para os estratos de renda de $3 \mathrm{SM}$, entre $3 \mathrm{SM}$ e 7 $\mathrm{SM}$ e maior que $7 \mathrm{SM}$, respectivamente. Os freezers reduziriam o consumo de $1,2 \%$ ao ano, $0,6 \%$ ao ano e $0,8 \%$ ao ano, seguindo a mesma ordem dos estratos de renda apresentados anteriormente.

No caso da posse desses equipamentos, foi utilizado um modelo logístico para fazer a projeção a partir das séries históricas disponibilizadas pelo IBGE (2012), partindo do pressuposto que há uma saturação da quantidade de equipamento por domicílio e isso é uma característica importante para adequação a este modelo. Para o estoque de geladeiras, a posse média por domicílio aumentaria, no período de 2005-2020, de 0,92 para 1,10 , para as famílias com renda até $3 \mathrm{SM}$; aumentaria de 1,02 para 1,06 para as famílias com renda entre 3 $\mathrm{SM}$ e $7 \mathrm{SM}$; e aumentaria de 1,08 para 1,09 para os domicílios com renda acima de 7 SM. Para o freezer, a posse média do estoque por domicílio aumentaria de 0,09 para 0,10 , reduziria de 0,21 para 0,19 e reduziria de 0,48 para 0,35 , no mesmo período e para os mesmos estratos de renda apresentados para a geladeira, respectivamente.

Condicionamento de ambientes:

Espera-se melhoria de eficiência dos aparelhos de ar condicionado por substituição de velhos equipamentos por novos, sendo $0,2 \%$ ao ano, para equipamentos existentes nos domicílios com renda até $7 \mathrm{SM}$, e 0,5\% ao ano para os demais equipamentos, sendo que, em 2020, não haveria nenhum equipamento com eficiência abaixo da faixa B, estabelecida pelo Inmetro (2011). Para os ventiladores, foi utilizada a mesma faixa de eficiência acima, sendo que todos os aparelhos existentes nos domicílios de renda até $7 \mathrm{SM}$ teriam uma redução de consumo de $1,1 \%$ ao ano e, para os demais equipamentos, a redução de consumo seria de $2,1 \%$ ao ano.

Para a projeção da posse dos aparelhos de ar condicionado e ventiladores, foi feito um ajuste logístico a partir dos dados da Pesquisa de Orçamentos Familiares (POF), elaborada pelo IBGE (1996), juntamente com os valores estimados a partir da pesquisa do Procel/Eletrobrás (2007), para o ano de 2005. Desta forma, considerou-se que para as famílias com renda até 3 SM o número de aparelhos de ar condicionado aumentaria de 7,5\% ao ano, de 20052010 , e $10,3 \%$ ao ano, para o restante do período; para as famílias com renda entre $3 \mathrm{SM}$ e $7 \mathrm{SM}$, aumentaria de $9,1 \%$ ao ano, no período de $2005-2010$, e $6,1 \%$ ao ano, para os demais anos e, finalmente, para as famílias com renda acima de 7 $\mathrm{SM}$, as taxas seriam de $2,8 \%$ ao ano e $1,8 \%$ ao ano, para o mesmo período dos outros estratos. Para os ventiladores, haveria aumento de $1,1 \%$ ao ano, período 
de 2005-2010, e 0,6\% ao ano, período de 2010-2020, para os ventiladores existentes nos domicílios de renda até 3 $\mathrm{SM}$, de $0,9 \%$ ao ano e $0,4 \%$ ao ano, nos mesmos períodos para o estrato de renda entre $3 \mathrm{SM}$ e $7 \mathrm{SM}$, e de $0,9 \%$ ao ano e $0,5 \%$ ao ano, para o estrato de renda acima de $7 \mathrm{SM}$.

Iluminação: Para a categoria de iluminação, considerou-se que em 2020 não haveria nenhuma lâmpada incandescente sendo utilizada para iluminação de ambientes, conforme Portaria $\mathrm{n}^{\mathrm{o}}$ 1.007/2010, do Ministério de Minas e Energia (Brasil, 2010b). Desta forma, todas as lâmpadas incandescentes seriam substituídas por lâmpadas fluorescentes compactas (FLC). Primeiramente, em 2015, não haverá lâmpadas incandescentes de $100 \mathrm{~W}$ e 150 $\mathrm{W}$ e, por isso, o consumo médio do estoque de lâmpadas seria de $47 \mathrm{kWh}$ ao ano para o estrato de renda até $3 \mathrm{SM}$, de $49 \mathrm{kWh}$ ao ano para o estrato de renda entre $3 \mathrm{SM}$ e $7 \mathrm{SM}$ e de $75 \mathrm{kWh}$ ao ano para o estrato de renda maior que $7 \mathrm{SM}$. No ano de 2020, o consumo será nulo para todos os estratos de renda. Também, foi considerado que o consumo do estoque de lâmpadas seria o mesmo de 2005 para cada estrato de renda, tanto para as lâmpadas fluorescentes compactas (FLC) quanto para as demais tecnologias. Com relação à projeção da posse, esperase que não haja grandes variações no número de lâmpadas por domicílios para cada estrato renda, como indica o PDE 2020 (Brasil, 2011), contudo, conforme Portaria $\mathrm{n}^{\circ} 1.007 / 2010$, haverá uma substituição de lâmpadas incandescentes por lâmpadas fluorescentes e demais tecnologias. Desta forma, neste trabalho foi considerada a mesma posse total (incandescentes e outras) para cada estrato durante o intervalo de projeção, sendo que a proporção de lâmpadas incandescentes diminuirá da seguinte maneira: de $71 \%$ em 2010 para $65 \%$ em 2015, chegando a 0\% em 2020 para o estrato de renda até $3 \mathrm{SM}$; de $60 \%$ em 2010 para $52 \%$ em 2015 e $0 \%$ em 2020 , para o estrato de renda entre $3 \mathrm{SM}$ e 7 SM, e; de $49 \%$ em 2010 para $40 \%$ em
2015 e $0 \%$ em 2020, para o estrato de renda acima de $7 \mathrm{SM}$.

Lazer: Dentre os equipamentos considerados nesta categoria, destaca-se o consumo do aparelho de TV e do computador. Os aparelhos de TVs são equipamentos de grande penetração nos domicílios brasileiros e de diferentes tamanhos e tecnologias como: CRT, LCD, Plasma e LEDs. Enquanto em 2005 a maioria dos aparelhos era de CRT e LCD, atualmente os aparelhos de CRT são minoria comparada com as demais tecnologias.

Se por um lado, a substituição tecnológica tem contribuído para reduzir o consumo de energia elétrica devido à penetração de equipamentos mais eficientes, por outro lado percebe-se que os novos equipamentos têm sido cada vez maiores e, portanto, aumentando significativamente o consumo de energia elétrica nesta categoria. Desta forma, foi considerado que os equipamentos existentes, em 2005, que foram agrupados por tamanho em quatro grupos: até 20 polegadas, de 20 e 21 polegadas, de 29 polegadas e maior que 30 polegadas, seriam substituídos até 2020 por equipamentos maiores, como se segue: 21 a 30 polegadas, 31 a 40 polegadas, 41 a 50 polegadas e maior que 50 polegadas, sucessivamente. Assim, haveria um aumento no consumo de energia elétrica de $3,3 \%$ ao ano, de $3,4 \%$ ao ano e $3,8 \%$ ao ano, para as famílias de renda de até 3 $\mathrm{SM}$; entre $3 \mathrm{SM}$ e $7 \mathrm{SM}$ e maior que 7 $\mathrm{SM}$, respectivamente. Estas taxas foram obtidas considerando o consumo médio do estoque de equipamento existente no mercado, conforme tabela do Inmetro (2011) e, também, utilizando a hipótese sugerida pelo PDE 2020, que propõe melhoria de eficiência dos aparelhos em 0,3\% ao ano (Brasil, 2011).

A projeção do consumo de energia para o computador está baseada na hipótese de que a frequência de uso mensal desses equipamentos deve aumentar devido ao maior acesso à internet. Portanto, a partir das taxas de crescimento do número de domicílios que utilizam o computador diariamente 
(Cetic, 2012), foi estimada a quantidade de dias por mês que as pessoas utilizam seus computadores para o ano de 2010 até 2020. Esses dados estão apresentados na Tabela 5, na sequência.

Tabelas 5 - Estimativas da utilização do computador para os três estratos de renda (total de dias por mês)

\begin{tabular}{ccccc}
\hline Renda & 2005 & $\mathbf{2 0 1 0}$ & $\mathbf{2 0 1 5}$ & $\mathbf{2 0 2 0}$ \\
\hline Até 3 SM & 11 & 15 & 20 & 24 \\
$\begin{array}{c}\text { Entre 3 e } \\
\text { 7SM }\end{array}$ & 13 & 16 & 18 & 20 \\
$\begin{array}{c}\text { Maior que } \\
\text { 7SM }\end{array}$ & 16 & 24 & 27 & 29 \\
\hline
\end{tabular}

Além disso, foi considerado que a potência dos computadores seria a mesma de 2005 e, também, o tempo de uso diário não mudaria para os três estratos de renda.

Para fazer as projeções de posse, também, foi utilizado um modelo logístico para os equipamentos pertencentes a esta categoria. A base de dados de alimentação para este modelo foi a POF (IBGE, 1996) e a PNAD (IBGE, 2012).

Serviço: Em 2005, o ferro elétrico foi o principal equipamento na categoria de Serviço. Somente ele foi responsável por $73 \%$ de toda energia consumida por esta categoria. Para projetar o consumo de energia para este equipamento, foi mantida a mesma potência, porém, considerando que devido à redução do número de pessoas por domicílio haveria também uma diminuição no uso deste equipamento. Desta forma, foram utilizadas as mesmas taxas de projeção do número de pessoas por domicílio para projetar o consumo de energia do ferro elétrico.

A projeção de consumo para a máquina de lavar seguiu a mesma lógica do ferro elétrico. Definido o consumo em 2005, este equipamento reduziria o consumo seguindo as mesmas taxas de projeção do número de pessoas por domicílio. A exceção é que para este equipamento foi considerada um melhoria na eficiência de $0,9 \%$ ao ano (Brasil, 2011).

O consumo de energia elétrica devido ao aparelho de micro-ondas é estimado da mesma forma que foi o dos demais equipamentos de sua categoria. Entretanto, considerou-se como hipótese que não haveria variação na projeção de consumo em relação a 2005. De fato, sabe-se que o consumo de energia pelo seu uso depende muito dos hábitos das famílias. Em regiões em que são consumidos muitos alimentos prontos (industrializados), o uso desse equipamento é maior que naquelas que possuem o hábito de consumir maior quantidade de alimentos "in natura". Também, pode-se afirmar que o uso do micro-ondas depende do peso da conta de energia elétrica no orçamento familiar e, portanto, a projeção de consumo de energia pelo uso do micro-ondas se baseia na hipótese de que tanto os hábitos regionais quanto o preço da energia e seu peso no orçamento não sofrerão significativas variações no horizonte de projeção.

A partir dos dados da POF (IBGE, 1996) e da PNAD (IBGE, 2012) foi projetada a posse para os equipamentos pertencentes a esta categoria. Para tanto, também, um modelo logístico foi utilizado.

Demais equipamentos: Apesar de esta categoria representar apenas $2 \%$ de toda energia elétrica consumida no setor residencial, em 2005, alguns estudos, incluindo o PDE 2020, apontam que ela será uma das principais categorias de uso final responsável pelo consumo de energia elétrica no futuro (Brasil, 2011). Isto pode ser justificado pelo aumento do número de ligações à rede elétrica, da 
melhoria da renda das famílias e principalmente pela penetração de equipamentos eletroeletrônicos como cooktops elétrico, panela elétrica, console de vídeo game, decodificador de sinal digital e tantos outros, em que suas posses e usos eram desprezíveis (até mesmo nula) nos lares brasileiros.

Devido às dificuldades de obtenção de informações sobre a posse e hábitos de uso para a maioria dos equipamentos nesta categoria foi preferível trabalhar com o consumo de energia de forma agregada, uma vez que o PDE 2020 apresenta projeções até 2020 para um conjunto de equipamentos e projeções de consumo de energia elétrica total para o setor residencial. Por isso, para este trabalho foi estimado o consumo desta categoria considerando o consumo em 2010 e a projeção de consumo de energia elétrica, em 2020, segundo PDE 2020 e subtraindo o consumo daqueles equipamentos que foram considerados tanto para 2010 quanto para 2020. Essa diferença, que representa o consumo de todos os equipamentos que não foram desagregados no PDE 2020, foi subtraída pelo consumo dos equipamentos que foram considerados nas categorias de Condicionamento de ambientes, Lazer e Serviço, neste artigo, e que também não foi contemplado pelo PDE 2020. O "resíduo" seria o consumo da categoria Demais equipamentos. A projeção da posse nesta categoria foi estimada, considerando a evolução das posses dos equipamentos conhecidos, mas não contemplados pelo PDE 2020, para cada estrato de renda.

\subsection{O método logarithmic mean divisia index}

Os métodos de análise de decomposição constituem uma metodologia utilizada para fazer análise e estudar os impactos das mudanças estruturais em um objeto de interesse, por exemplo, mudanças de intensidade energética em um dado setor. Eles têm sido utilizados desde o início da década de 1970, principalmente no setor industrial. Desde então, vários estudos foram feitos e em diferentes setores com diferentes métodos e objetivos (Achão, 2009).

Dentre os métodos mais utilizados, podem-se citar os métodos baseados no Divisia Index e os métodos derivados do índice de Laspeyres. Da família Divisia Index, podem-se citar os métodos Divisia de Média Aritmética (AMDI) e Divisia de média Logarítmica (LMDI) e, da família Laspeyres, podemse citar os métodos Paasche e MarshallEdgeworth (Wachsmann, 2005).

Dentre os diversos métodos, o Logarithmic Mean Divisia Index (LMDI) é um dos métodos mais recomendados para fazer análise de decomposição energética, porque ele apresenta um conjunto de vantagens quando comparado com outros métodos, que são: simplicidade, decomposição perfeita (não produz resíduo) e possui uma consistência na agregação dos termos decompostos (Ang \& Liu, 2001; Ang, 2004, 2005). Pelos motivos citados, esta metodologia é utilizada neste artigo.

No Brasil, poucos trabalhos foram desenvolvidos utilizando essas técnicas de análise de decomposição, sendo eles a maioria para análise do setor industrial (Rosa e Tolmasquim, 1993; Worrell et al., 1997; Machado e Schaeffer, 2006). Desta forma, foram raros os trabalhos produzidos para outros setores. Em especial para o setor residencial, que é o setor considerado neste artigo, podem ser citados Wachsmann (2005), Leon e Pessanha (2005) e Achão (2009). Este último foi a principal referência para o desenvolvimento deste artigo, uma vez que este utilizou a técnica de decomposição LMDI para avaliar a evolução do consumo de energia, a partir da variação na participação do número de consumidores em cada região geográfica com relação ao total no país e, também, na variação do número de consumidores de baixa renda e convencionais com relação ao total no Brasil. Já neste artigo, a análise de decomposição é utilizada para avaliar a evolução de consumo de energia elétrica no setor residencial a 
partir da variação na posse de equipamentos eletroeletrônicos, da variação no consumo específico desses equipamentos e da variação na participação do número de consumidores por renda com relação ao total no Brasil.

\subsection{A formulação do LMDI e sua aplicação no setor residencial}

Considere que E seja a energia total de um dado setor e que ela possa ser desagregada em $\mathbf{x}_{\mathbf{i j}}$ variáveis, em que $\mathbf{j}$ representa os tipos de equipamentos eletroeletrônicos e i representa os fatores de desagregação, conforme apresentado a seguir:

$$
E=\sum_{j=1}^{m} \prod_{i}^{n} x_{i j}
$$

sendo, para um dado tempo inicial, $\mathrm{T}_{0} \mathrm{e}$, para um período posterior, $\mathrm{T}$ :

$$
\begin{aligned}
& E^{T_{0}}=\sum_{j=1}^{m} \prod_{i}^{n} x^{T_{0}}{ }_{i j} \\
& E^{\mathrm{T}}=\sum_{j=1}^{m} \prod_{i}^{n} x^{T}{ }_{i j}
\end{aligned}
$$

Segundo Ang e Liu (2001) e Ang (2004; 2005), a metodologia LMDI na forma multiplicativa da decomposição pode ser escrita:

$$
\mathrm{D}_{\text {total }}=E^{\mathrm{T}} / E^{T_{0}}=\prod_{i}^{n} D_{x_{\hat{i}}}
$$

Em que o fator k-ésimo é:

$$
D_{x_{k}}=\exp \left[\sum_{j}^{m} \frac{L\left(E_{j}^{T}, E_{j}^{T_{0}}\right)}{L\left(E^{T}, E^{T_{0}}\right)} \ln \left(\frac{x_{k, j}^{T}}{x_{k, j}^{T_{0}}}\right)\right]
$$

$L(a, b)=(a-b) / \ln (a)-\ln (b), \quad$ para $\mathrm{a} \neq \mathrm{b}$, $L(a, b)=a, \quad$ para $\mathrm{a}=\mathrm{b}$.

$\mathrm{Na}$ forma aditiva pode ser escrita:

$$
\begin{gathered}
\Delta \mathrm{E}_{\mathrm{total}}=E^{T}-E^{T_{0}}=\sum_{i}^{n} \Delta E x_{i} \\
\Delta \mathrm{E} x_{k}=\sum_{j}^{m} L\left(E_{j}^{T}, E_{j}^{T_{0}}\right) \ln \left(\frac{x_{k j}^{T}}{x_{k, j}^{T 0}}\right)
\end{gathered}
$$

É importante observar que há uma relação entre a forma aditiva e multiplicativa de LMDI que pode ser escrita como:

$$
\Delta \mathrm{E} / \ln (\mathrm{D})=L\left(E^{T}, E^{T_{0}}\right)
$$

Portanto, tanto faz utilizar a forma aditiva ou a forma multiplicativa no processo de decomposição. Neste trabalho será utilizada a forma aditiva por ser mais simples.

Para aplicar tal metodologia neste estudo, a energia elétrica consumida no setor residencial (E) foi desagregada como segue:

$$
\mathrm{E}=\sum_{j}^{m} \sum_{i}^{n} Q \frac{Q_{j}}{Q} \frac{q_{i j}}{Q_{j}} \frac{c_{i j}}{q_{i j}},
$$

Em que $Q$ é a quantidade de domicílios consumidores, $\mathrm{Q}_{\mathrm{j}}$ é a quantidade de domicílios consumidores em um estrato de renda (j), $\mathrm{q}_{\mathrm{ij}}$ é a quantidade de um dado equipamento (i) no estrato de renda (j) e $\mathrm{C}_{\mathrm{ij}}$ é o consumo de energia elétrica total para um equipamento específico (i) no estrato de renda (j).

$\mathrm{Na}$ equação 9, a razão $\mathrm{Q}_{\mathrm{j}} / \mathrm{Q}$ corresponde à quantidade de domicílios consumidores em um estrato de renda (j) relativo ao total de domicílios consumidores, sendo o quociente representado por $\left(\mathrm{S}_{\mathrm{j}}\right) ; \mathrm{q}_{\mathrm{ij}} / \mathrm{Q}_{\mathrm{j}}$ representa a posse de um equipamento específico (i) por domicílio consumidor no estrato de renda $(\mathrm{j})$, sendo o quociente representado por $\left(\mathrm{P}_{\mathrm{ij}}\right)$ e $\mathrm{C}_{\mathrm{ij}} / \mathrm{q}_{\mathrm{ij}}$ pode ser entendida como a intensidade de um tipo de equipamento (i) no estrato de renda (j), ou simplesmente, consumo específico para um equipamento (i) no estrato de renda (j), sendo o quociente representado por $\left(\mathrm{I}_{\mathrm{ij}}\right)$.

Assim, o consumo de eletricidade no setor residencial pode ser escrito em termos do efeito Atividade (Q), do efeito Estrutural (S), o efeito Posse (P) e em termos do efeito Intensidade (I):

$$
\mathrm{E}=\sum_{j}^{m} \sum_{i}^{n} Q S_{j} P_{i j} I_{i j}
$$


Neste caso, somam-se os efeitos dos equipamentos $i$ até $n$ e também, somamse os estratos de renda $j$ até $m$.

Por exemplo, o efeito Posse na variação de consumo de eletricidade de um késimo equipamento pode ser escrito como:

$$
\Delta \mathrm{E}\left(P_{k}\right)=\sum_{j}^{\mathrm{m}}\left(\frac{E_{k j}^{T}-E_{k j}^{T_{0}}}{\ln E_{k j}{ }^{T}-\ln E_{k j}{ }^{T 0}}\right) \ln \frac{p_{k}^{T}}{p_{k 0}^{T 0}}(11)
$$

\section{Análise dos resultados}

$\mathrm{Na}$ Tabela 6 são apresentadas as projeções de consumo específico, por equipamento, para o ano 2020.

Tabela 6 - Projeções do consumo específico a partir do estoque de eletroeletrônicos, em 2020 (kWh/ano)

\begin{tabular}{|c|c|c|c|}
\hline Equipamentos & Até 3SM & $\begin{array}{c}\text { Entre } \\
3 S M \\
\text { e } \\
7 S M\end{array}$ & $\begin{array}{c}\text { Maior } \\
\text { que } \\
7 S M\end{array}$ \\
\hline Chuveiro elétrico & 468 & 682 & 871 \\
\hline Geladeira & 352 & 441 & 440 \\
\hline Ar condicionado & 251 & 414 & 445 \\
\hline Lâmpada incandescente & - & - & - \\
\hline Lâmpada fluorescente & 19 & 18 & 28 \\
\hline Freezer & 291 & 363 & 463 \\
\hline Aparelho de Televisão & 159 & 187 & 205 \\
\hline M. Lava roupa & 8 & 10 & 12 \\
\hline Micro-ondas & 60 & 68 & 83 \\
\hline Ferro de passar roupa & 73 & 91 & 107 \\
\hline Aparelho de som & 44 & 47 & 55 \\
\hline Computador & 46 & 44 & 87 \\
\hline Ventilador de mesa & 25 & 33 & 33 \\
\hline Ventilador de teto & 25 & 34 & 34 \\
\hline
\end{tabular}

Comparando com o consumo de energia elétrica, em 2005, percebe-se que, para a maioria dos equipamentos, o consumo específico reduzirá, exceto para os aparelhos de televisão, ferro de passar roupa e computadores, que apresentam um aumento no consumo, e para as lâmpadas fluorescentes, os aparelhos de micro-ondas e os aparelhos de som, que por hipótese foi mantido constante. No caso do aparelho de televisão, a compra de novos aparelhos com tamanhos de telas maiores seria um dos principais responsáveis pelo aumento de consumo de energia para este equipamento. No caso dos computadores, o aumento do acesso à internet será o principal responsável pelo aumento do consumo específico para este equipamento.

$\mathrm{Na}$ Tabela 7 são apresentadas as projeções da posse, por equipamento, para o ano 2020.

Tabela 7 - Projeções da posse de equipamentos eletroeletrônicos, por domicilio, no Brasil, em 2020

\begin{tabular}{ll|cc}
\hline \multicolumn{1}{c}{ Equipamentos } & Até 3SM & $\begin{array}{c}\text { Entre } \\
\mathbf{3} \text { e }\end{array}$ & $\begin{array}{c}\text { Maior } \\
\text { que }\end{array}$ \\
& & $\mathbf{7 S M}$ & $\mathbf{7 S M}$ \\
\hline Chuveiro elétrico & 0,50 & 0,77 & 0,80 \\
\hline Geladeira & 1,10 & 1,06 & 1,09 \\
\hline Ar condicionado & 0,07 & 0,25 & 0,58 \\
\hline Lâmpada incandescente & 0,00 & 0,00 & 0,00 \\
\hline Lâmpada fluorescente & 5,74 & 8,04 & 12,4 \\
\hline Freezer & 0,10 & 0,19 & 0,35 \\
\hline Aparelho de Televisão & 1,18 & 1,42 & 2,03 \\
\hline M. Lava roupa & 0,56 & 0,89 & 1,00 \\
\hline Micro-ondas & 0,15 & 0,40 & 0,70 \\
\hline Ferro de passar roupa & 0,90 & 1,00 & 1,01 \\
\hline Aparelho de som & 0,63 & 0,87 & 1,14 \\
\hline Computador & 0,80 & 0,94 & 0,91 \\
\hline Ventilador de mesa & 0,68 & 0,88 & 1,01 \\
\hline Ventilador de teto & 0,18 & 0,52 & 0,79 \\
\hline
\end{tabular}

Nesta Tabela 7, vale destacar que a posse de lâmpadas incandescentes é nula porque conforme a Portaria $\mathrm{n}^{\circ}$ 1.007/2010, do Ministério de Minas e Energia (Brasil, 2010b), essas lâmpadas deixarão de ser produzidas e comercializadas antes de 2020.

$\mathrm{Na}$ Tabela 8 são apresentadas as projeções de consumo de energia elétrica no setor residencial, por categoria de uso final, para os três estratos de renda, no Brasil.

Tabela 8 - Consumo de energia elétrica no setor residencial, por categoria, em 2020 (TWh/ano)

\begin{tabular}{ccccc}
\hline Categorias & Até & Entre & Maior & Total \\
& $3 S \mathrm{M}$ & $3 \mathrm{M} \mathrm{e}$ & que & \\
\hline
\end{tabular}




\begin{tabular}{ccccc}
\hline & & $\mathbf{7 S M}$ & $\mathbf{7 S M}$ & \\
\hline Aquecimento de & 7,3 & 14,6 & 11,8 & 33,6 \\
água & $(17 \%)$ & $(22 \%)$ & $(17 \%)$ & $(19 \%)$ \\
Condicionamento & 13,0 & 14,9 & 10,9 & 38,8 \\
de alimentos & $(30 \%)$ & $(23 \%)$ & $(16 \%)$ & $(22 \%)$ \\
Condicionamento & 1,2 & 4,2 & 5,4 & 10,7 \\
de ambientes & $(3 \%)$ & $(6 \%)$ & $(8 \%)$ & $(6 \%)$ \\
lluminação & 3,4 & 4,0 & 5,9 & 13,3 \\
& $(8 \%)$ & $(6 \%)$ & $(9 \%)$ & $(8 \%)$ \\
Serviço & 2,5 & 3,6 & 3,0 & 9,0 \\
& $(6 \%)$ & $(5 \%)$ & $(4 \%)$ & $(5 \%)$ \\
Lazer & 7,8 & 9,7 & 9,5 & 26,9 \\
Demais & $(18 \%)$ & $(15 \%)$ & $(14 \%)$ & $(15 \%)$ \\
equipamentos & 8,1 & 14,9 & 21,2 & 44,3 \\
& $(19 \%)$ & $(23 \%)$ & $(31 \%)$ & $(25 \%)$ \\
Total & $\mathbf{4 3 , 4}$ & $\mathbf{6 5 , 9}$ & $\mathbf{6 7 , 6}$ & $\mathbf{1 7 6 , 6}$ \\
& $(\mathbf{1 0 0 \% )}$ & $\mathbf{( 1 0 0 \% )}$ & $\mathbf{( 1 0 0 \% )}$ & $\mathbf{( 1 0 0 \% )}$ \\
\hline
\end{tabular}

Conforme a Tabela 8, a projeção de consumo de energia elétrica no setor residencial para o Brasil, em 2020, será de 177 TWh, para um crescimento do PIB de 5,0\% ao ano. Este valor representa um aumento de $110 \%$ com relação ao consumo de 2005.

Comparando com a Tabela 3, pode-se dizer que apenas para a categoria de Iluminação e para a categoria de Aquecimento de água (até $3 \mathrm{SM}$ ) haverá uma redução no consumo de energia em comparação a 2005. Como já foi dito, a proibição da fabricação e venda de lâmpadas incandescentes reduzirá o consumo na categoria Iluminação, enquanto a substituição do chuveiro elétrico por aquecedores solares, principalmente nos domicílios de baixa renda, será responsável pela redução de energia na categoria de Aquecimento de água. Para as demais categorias haverá aumento no consumo de energia elétrica.

Estes resultados foram baseados nas hipóteses de que o PIB nacional crescerá $5,0 \%$ ao ano e de que haverá uma melhor distribuição de renda. Entretanto, a economia brasileira não tem crescido como se esperava. De acordo com World Economic Outlook, elaborado pelo Fundo Monetário Internacional (FMI), em 2013, o PIB nacional neste ano crescerá 3,0\% e em 2014 crescerá 4,0\% (FMI, 2013). Diante deste quadro econômico, foram feitas alterações na projeção de consumo de energia elétrica (elaborado anteriormente), considerando que o PIB nacional seja de $4,0 \%$ ao ano até o final do horizonte de projeção e que a elasticidade-renda do consumo de energia elétrica no setor residencial seja a mesma estimada no PDE 2020 (Brasil, 2011). Também, foi considerada uma pior distribuição de renda comparada com este cenário. Neste caso, por hipótese, o estrato de renda até $3 \mathrm{SM}$ será $10 \%$ maior, a proporção de domicílios no estrato de renda entre $3 \mathrm{SM}$ e 7 SM será a mesma e no estrato maior que $7 \mathrm{SM}$ será menor. Isto implica que $45,0 \%$ dos domicílios consumidores existentes no Brasil, em 2020, estariam no estrato de renda até $3 \mathrm{SM}$ e apenas 18,3\% dos domicílios estariam no estrato de renda maior que 7SM, ficando o restante dos domicílios no estrato entre $3 \mathrm{SM}$ e $7 \mathrm{SM}$. A mudança destas duas variáveis socioeconômicas no cenário permite fazer uma análise de sensibilidade do consumo de energia elétrica. A Figura 2 mostra qual seria a variação de consumo de energia elétrica, caso o PIB seja menor que o projetado no cenário considerado neste estudo e caso a distribuição de renda seja menos igualitária.

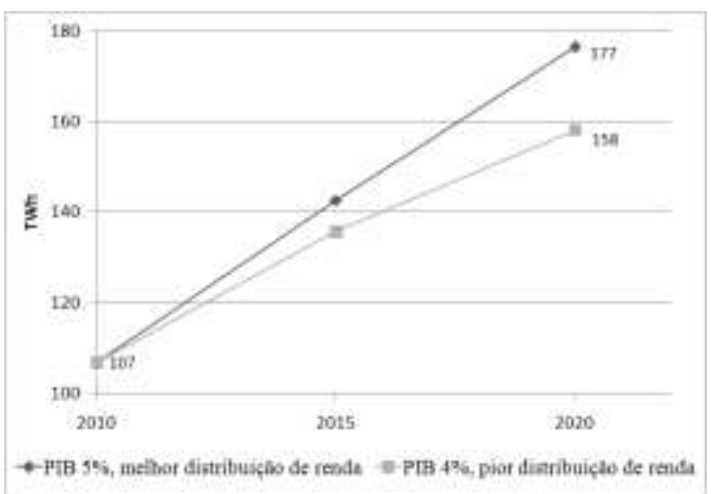

Figura 2: Comparação das projeções de consumo de energia elétrica para o cenário com maior PIB versus menor PIB.

Conforme mostra a Figura 2, a diferença de consumo de energia elétrica no setor residencial seria de $19 \mathrm{TWh}$, em 2020, ou seja, 10,7\% com relação ao cenário com o PIB maior. Este resultado mostra que levando em consideração apenas diferenças em duas variáveis socioeconômicas, a variação no consumo de energia é mais representativa que, por exemplo, a redução do consumo de energia no setor residencial apresentado pelo Plano Decenal de Expansão de 
Energia (PDE2020). Segundo este relatório, em 2020, a conservação de energia elétrica no setor residencial será de 6,8 TWh devido à melhoria da eficiência energética neste setor (Brasil, 2011).

Aplicando a metodologia análise de decomposição para as projeções de consumo de energia elétrica, percebe-se que o efeito Atividade será responsável por $57 \%$ da variação de consumo no período de 2005-2020, conforme mostra a Figura 3. Nesta figura é mostrada a decomposição da variação de consumo pelos quatros efeitos; Atividade, Estrutura, Intensidade e Posse, no período de 2005-2020.

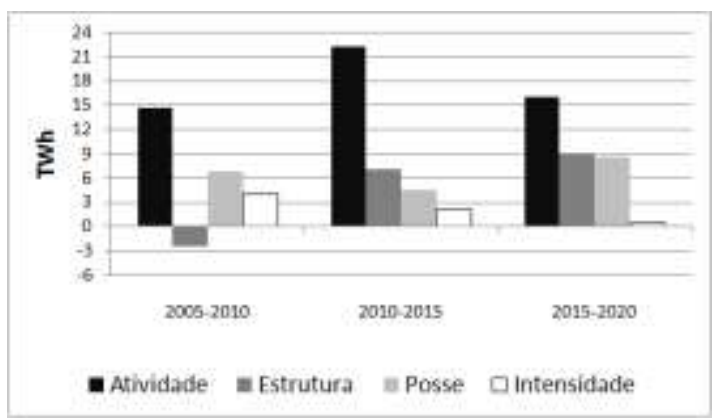

Figura 3: Projeções do consumo de energia elétrica desagregado por renda e efeito

O efeito Atividade está associado ao número de domicílios e, portanto, os resultados indicam que o principal motivo do aumento do consumo de energia elétrica no setor residencial (conforme projeções) estará ligado ao crescimento do número de domicílios com acesso à energia elétrica. Também, vale destacar que a redução do número de famílias (efeito Estrutura) que possuem renda até 3 SM contribuirá com 10,9 TWh para a redução de consumo de energia elétrica, no período de 2005-2020, e a variação do número das famílias com renda maior que 7 SM aumentará o consumo em 14,1 TWh. Totalizando um efeito Estrutura positivo de 13,4 TWh para todas os estratos de renda.

Na Tabela 9 é apresentado o efeito Intensidade para todos os estratos de renda e para as categorias de uso final, no período 2005-2020.
Tabela 9 - Efeito Intensidade desagregado por renda, no período 2005-2020 (TWh)

\begin{tabular}{lccc}
\hline Categorias de uso final & $\begin{array}{c}\text { Até 3 } \\
\text { SM }\end{array}$ & $\begin{array}{c}\text { Entre } \\
\text { 3 e 7 } \\
\text { SM }\end{array}$ & $\begin{array}{c}\text { Maior } \\
\text { que 7 } \\
\text { SM }\end{array}$ \\
\hline $\begin{array}{l}\text { Aquecimento de água } \\
\begin{array}{l}\text { Condicionamento de } \\
\text { alimentos }\end{array}\end{array}$ & $-1,04$ & $-0,58$ & $-0,33$ \\
\hline $\begin{array}{l}\text { Condicionamento de } \\
\text { ambientes }\end{array}$ & $-0,12$ & $-0,20$ & $-0,44$ \\
\hline Iluminação & $-3,10$ & $-2,81$ & $-2,86$ \\
\hline Serviço & 0,16 & 0,15 & 0,09 \\
\hline Lazer & 2,45 & 2,37 & 2,34 \\
\hline Demais equipamentos & 3,31 & 4,66 & 6,10 \\
\hline Total & 0,01 & 3,17 & 3,52 \\
\hline
\end{tabular}

A partir da Tabela 9, pode-se perceber que as categorias de Aquecimento de água, Condicionamento de alimentos, Condicionamento de ambientes e Iluminação deverão reduzir seus consumos de energia elétrica, seja por substituição de tecnologia, como é o caso do chuveiro elétrico por aquecimento solar e a gás, seja por melhoria de eficiência, como é o caso das geladeiras e aparelhos de ar condicionado. Contudo, as demais categorias mostram um aumento no consumo de energia. Sendo que isto deverá ser devido à aquisição de equipamentos mais potentes, como é o caso da máquina de lavar roupa, e mudanças de hábitos de uso, como é o caso do uso do computador devido à popularização da internet.

Já na Tabela 10 é apresentado o efeito Posse na variação de consumo de energia elétrica para as categorias de uso final, também desagregado por renda e no mesmo período.

Tabela 10 - Efeito Posse para cada categoria de uso final desagregado por renda no período 2005-2020 (TWh)

\begin{tabular}{llll}
\hline Categorias de uso final & $\begin{array}{l}\text { Até } \\
\text { 3M }\end{array}$ & $\begin{array}{l}\text { Entre } \\
\mathbf{3} \text { e 7 } \\
\text { SM }\end{array}$ & $\begin{array}{l}\text { Maior } \\
\text { que 7 } \\
\text { SM }\end{array}$ \\
\hline $\begin{array}{l}\text { Aquecimento de água } \\
\begin{array}{l}\text { Condicionamento de } \\
\text { alimentos }\end{array}\end{array}$ & $-1,02$ & $-0,46$ & $-0,24$ \\
$\begin{array}{l}\text { Condicionamento de } \\
\text { ambientes }\end{array}$ & 2,26 & 0,21 & $-0,62$ \\
\hline
\end{tabular}




\begin{tabular}{lccc}
\hline Iluminação & $-1,06$ & $-1,00$ & $-0,97$ \\
Serviço & 0,32 & 0,20 & 0,07 \\
\hline Lazer & 1,36 & 0,82 & 0,39 \\
Demais equipamentos & 4,26 & 5,56 & 6,81 \\
\hline Total & 6,58 & 6,80 & 6,30 \\
\hline
\end{tabular}

Como dito anteriormente, a Portaria $\mathrm{n}^{\circ} 1.007 / 2010$, do Ministério de Minas e Energia, que tem limitado a fabricação e venda de lâmpadas incandescentes gerará uma redução significativa do consumo de energia elétrica. Conforme mostram as Tabelas 9 e 10, a substituição de lâmpadas incandescentes por fluorescentes compactas será a responsável por uma redução de 11,8 TWh para o efeito Intensidade e Posse no período de 20052020.

A categoria Demais equipamentos será a principal categoria responsável pelo aumento de energia elétrica no setor residencial tanto para o efeito Intensidade quanto para o efeito Posse. E isso se deve, principalmente, à expectativa de melhoria da renda das famílias e à facilidade de compra de novos equipamentos eletroeletrônicos devido à tendência de redução de juros para as compras a prazo. Ao finalizar esta análise, deve-se observar que a projeção de consumo de energia elétrica realizada neste artigo não considerou alguns aspectos importantes associados às premissas demográficas. Para projetar o número de domicílios consumidores foram consideradas as taxas elaboradas pelo PDE2020, as quais são baseadas em uma metodologia de extrapolação linear da densidade domiciliar. Contudo, como aponta Pessanha e Leon (2012) esta técnica não leva em consideração a transição da estrutura etária da população brasileira que pode produzir efeitos distintos da extrapolação linear. Segundo estes autores, a demanda de energia elétrica per capita no setor residencial deverá aumentar com o envelhecimento da população e deverá diminuir devido ao aumento do número de domicílios com menor densidade de moradores.

\section{Conclusões}

As projeções de consumo de energia elétrica no setor residencial mostram que o consumo deverá aumentar $110 \%$, no período de 2005-2020, ou seja, o consumo de energia elétrica vai dobrar em apenas 15 anos.

Com relação à metodologia de análise de decomposição, aplicada às projeções de consumo de energia, mostrou-se uma ferramenta bastante eficaz para indicar qual efeito, ou categoria ou estrato de renda poderá aumentar o consumo mais significativamente. Fica como sugestão a utilização da metodologia Logarithmic Mean Divisia Index (LMDI) nos planos nacionais.

$\mathrm{Na}$ análise dos efeitos Atividade, Intensidade e Posse, o primeiro mostrouse que será o maior responsável pelo aumento no consumo de energia elétrica. Somente o efeito Atividade representará $57 \%$ do aumento no consumo de energia, no período 2005-2020.

Outro ponto de destaque nesta pesquisa é o dimensionamento do efeito da política de eficiência energética para a iluminação. A substituição total de lâmpadas incandescentes por fluorescentes compactas será responsável por uma redução de $11,8 \mathrm{TWh}$ somente para o efeito Intensidade e Posse. Este valor representa quase $7 \%$ do consumo de energia elétrica em 2020.

Esses resultados mostram que são necessárias políticas sólidas de eficiência e conservação de energia no setor residencial, uma vez que o consumo de energia elétrica no setor residencial brasileiro poderá aumentar $110 \%$ de 2005 até 2020, sendo que o aumento do número de domicílios e aquisição de equipamentos que em 2005 basicamente não existiam nos lares brasileiros seriam os principais responsáveis por este aumento de consumo. Portanto, as políticas de conservação e eficiência energética para o setor residencial devem contemplar todas as categorias de uso final, juntamente com todas as variáveis que influenciam o consumo, inclusive o 
próprio consumidor, agente fundamental da demanda futura de energia no setor residencial.

\section{REFERÊNCIAS}

ACHÃO, C. C. L. 2009. Análise de Decomposição das Variações no Consumo de Energia Elétrica no Setor Residencial Brasileiro. Tese de D. Sc., COPPE/UFRJ. Rio de Janeiro.

ANG, B. W. Decomposition analysis for policymaking in energy: which is the preferred method. Energy Policy 32, 1131-1139, 2004.

ANG, B. W. The LMDI approach to decomposition analysis: a pratical guide. Energy Policy 33, 867-871, 2005.

ANG, B. W.; Liu, F. L. A new energy decomposition method: perfect in decomposition and consistent in aggregation. Energy 26, 537-548, 2001.

ARAUJO, A. C. M. 2007. Perdas e inadimplência na atividade de distribuição de energia elétrica no Brasil. 98p. Tese de doutorado em Sc.; Planejamento Energético, COPPE/ UFRJ. Rio de Janeiro.

Brasil. Ministério de Minas e Energia. EPE, Empresa de Pesquisa Energética. Avaliação da Eficiência Energética na Indústria e nas Residências no Horizonte Decenal 2010-2019 (nota técnica). Rio de Janeiro, 2010a. Disponível em http://www.epe.gov.br/mercado/Docume nts/Séries $\quad$ Estudos de Energia/20100809 4.pdf. Acessado em abril/ 2013

Brasil. Ministério de Minas e Energia. Portaria Interministerial $n^{\circ} 1.007$ de 31 de dezembro de 2010. Brasília/DF, $2010 b$.

Brasil. Ministério de Minas e Energia/Empresa de Pesquisa Energética. Plano Decenal de Expansão de EnergiaPDE-2020, Brasília, 2011.

Brasil. Ministério de Minas e Energia. Empresa de Pesquisa Energética. Balanço Energético Nacional-BEN2012: ano base 2011. Rio de Janeiro: EPE, 2012a.
Brasil. Ministério das Cidades. Programa Minha Casa Minha Vida, fase 1 e 2. Brasília/DF, 2012b. Disponível em http://downloads.caixa.gov.br/_arquivos/ habita/mcmv/CARTILHACOMPLETA.P DF. Acessado em julho/ 2012.

BRASIL, Portal Brasil. Brasília/DF, 2013. Disponível em http://www.brasil.gov.br/infraestrutura/20 13/09/mais-de-183-mil-casas-temaquecimento-solar-no-Brasil. Acessado em outubro/ 2013.

Centro de Estudos Sobre as Tecnologias da Informação e da Comunicação. Pesquisa sobre o uso das tecnologias da informação e comunicação no Brasil. Ano de 2005 a 2011. São Paulo. Cetic, 2012. Disponível em http://www.cetic.br/usuarios/tic/.Acessad o em setembro/ 2012.

Ernst \& Young Terco. Brasil Sustentável: Crescimento econômico e potencial de consumo. Fundação Getúlio Vargas, 2008. Disponível em http://www.ey.com/Publication/vwLUAss ets/Crescimento_Econômico_e_Potencial _de_Consumo_PDF_Publicação/\$FILE/C rescimento_Econômico_e_Potencial_de Consumo.pdf. Acessado em Dezembro/ 2012.

Fundo monetário internacional. World Economic Outlook: Hopes, Realities and Risks, abril de 2013. Washington, EUA. FMI, 2013. Disponível em http://www.imf.org/external/pubs/ft/weo/ 2013/01. Acessado em junho/ 2013.

Instituto Brasileiro de Geografia e Estatística. Pesquisa de Orçamentos Familiares (POF). Rio de Janeiro, Brasil. IBGE,1996.

Instituto Brasileiro de Geografia e Estatística. Pesquisa Nacional por Amostra de Domicílios (PNAD) -2009. Brasil. IBGE, 2009.

Instituto Brasileiro de Geografia e Estatística. Pesquisa Nacional por Amostra de Domicílios (PNAD). Banco de dados agregados- SIDRA. Brasil. IBGE, 2012. 
Instituto Nacional de Metrologia, Normalização e Qualidade Industrial. Tabelas de consumo/eficiência energética, Produtos. Inmetro, 2011. Disponível em http://www.inmetro.gov.br/consumidor/ta belas.asp. Acessado em novembro/ 2011.

LEON, N., PESSANHA, J.F.M. Dinâmica da evolução do consumo de energia no setor residencial. Anais do XVIII SNPTEE - Seminário Nacional de Produção e Transmissão de Energia Elétrica. Curitiba, Brasil, 2005.

MACHADO, G., SCHAEFFER, R. Energy and Economic Development. In: Brazil: A Country Profile on Sustainable Energy Development. (International Atomic Energy Agency, et al.). Vienna: IAEA, 2006.

PESSANHA, J.F.M, Leon, N. Long-term forecasting of household and residential electric customers in Brazil. IEEE America Latina 10, 1537-1543, 2012.

Programa Nacional de Conservação de Energia/Eletrobrás. Pesquisa de Posse de Eletrodomésticos e Hábitos de Uso -
Setor Residencial (Relatório Brasil). Rio de Janeiro. Procel/Eletrobrás, 2007.

Programa Nacional de Conservação de Energia/Eletrobrás. Tabela de estimativa de consumo médio mensal de eletrodomésticos - 2011. Rio de Janeiro. Procel/Eletrobrás, 2011. Disponível em http://www.eletrobras.com/elb/procel/mai n.asp. Acessado em agosto/ 2011.

ROSA, L.P., TOLMASQUIM, M.T. An analytical model to compare energy efficiency indices and $\mathrm{CO} 2$ emissions in developed and developing countries. Energy Policy 21, 276-283, 1993.

WORRELL, E., Price, L., Martin, N., Farla, J., Schaeffer, R. Energy intensity in the iron and steel industry: a comparison of physical and economic indicators. Energy Policy 25, 727-744, 1997.

WACHSMANN, U., 2005. Mudanças no Consumo de Energia e nas Emissões Associadas de $\mathrm{CO}_{2}$ no Brasil entre 1970 e 1996. Uma Análise de Decomposição Estrutural. Tese de D. Sc., COPPE/UFRJ, Rio de Janeiro, Brasil. 